\title{
Lion Feuchtwanger : le philologue et les cochons
}

Une dénonciation de la manipulation de l'irrationnel et du mythe par les nazis

Lion Feuchtwanger: der Philologe und die Schweine. Eine Entlarvung der Manipulation des Irrationalen und des Mythos durch die Nazis

Lion Feuchtwanger: the Philologist and the Pigs. A Denunciation of the

Manipulation of the Irrational and the Myth by the Nazis

\section{Frédéric Teinturier}

\section{(2) OpenEdition}

\section{Journals}

Édition électronique

URL : http://journals.openedition.org/ceg/4373

DOI : $10.4000 /$ ceg.4373

ISSN : 2605-8359

\section{Éditeur}

Presses Universitaires de Provence

Édition imprimée

Date de publication : 2 mai 2019

Pagination : 81-94

ISBN : 979-10-320-0214-8

ISSN : 0751-4239

\section{Référence électronique}

Frédéric Teinturier, «Lion Feuchtwanger : le philologue et les cochons », Cahiers d'Études Germaniques [En ligne], 76 | 2019, mis en ligne le 02 novembre 2020, consulté le 25 janvier 2021. URL : http:// journals.openedition.org/ceg/4373; DOI : https://doi.org/10.4000/ceg.4373 


\section{Lion Feuchtwanger : le philologue et les cochons Une dénonciation de la manipulation de l'irrationnel et du mythe par les nazis}

Frédéric TEINTURIER

Université de Lorraine (Metz), CEGIL (EA 3944)

\section{Introduction}

L'étude qui suit porte sur le roman Die Brüder Lautensack et sur le récit Odysseus und die Schweine oder das Unbehagen an der Kultur de Lion Feuchtwanger. Une précision est nécessaire sur le choix de rapprocher les deux textes. Au-delà des aspects thématiques - le véritable sujet du roman et de la nouvelle consiste pour Feuchtwanger à montrer la fragilité du sens d'un texte littéraire -, le contexte d'écriture les rapproche également. En effet, ces deux textes ont été écrits par le romancier durant son exil américain ${ }^{1}$. L'étude adopte un positionnement scientifique volontairement centré sur l'analyse philologique, dans le but d'interpréter le sens parfois problématique car non immédiat de ces textes narratifs; cette démarche met en avant la notion de lecture et d'interprétation. Dans la mesure où les textes étudiés sont certes connus mais n'ont, à notre connaissance, quasiment pas été l'objet d'analyses interprétatives précises ${ }^{2}$, il semble essentiel de les soumettre à cette interrogation philologique d'autant plus primordiale que, justement, ces récits de Feuchtwanger se caractérisent par un jeu philologique du narrateur avec son lecteur, le premier invitant le second à entrer dans une réflexion interprétative sur le sens des

1. Le roman a été publié en anglais (The Brothers Lautensack, London, Hamish Hamilton, 1943) puis, la même année, en allemand (Die Brüder Lautensack, London, Hamish Hamilton, 1943), sans que des changements notables interviennent entre les deux versions. En revanche, Feuchtwanger avait d'abord pensé, en 1941, faire de ce sujet une pièce de théâtre; ce projet, inabouti, était fort différent. Quant au récit Odysseus, il a certes été composé en 1947 (cf. infra, note 24), mais par un auteur qui n'envisageait pas, en tout cas à court terme, de retour en Allemagne. C'est donc bien encore l'œuvre d'un exilé, c'est-à-dire d'un écrivain qui vit dans un pays étranger. Même si le nazisme a disparu au moment où il écrit la nouvelle, cela ne change rien à la posture de Feuchtwanger et à sa critique des manipulateurs du sens des textes. Dans le roman, ces manipulateurs sont les nazis, dans la nouvelle, le propos n'est pas directement politique.

2. C'est la raison pour laquelle on trouvera ici peu de références critiques : cet aspect de son œuvre est peu étudié. Les textes analysés ici sont le plus souvent rapidement mentionnés au sein d'études plus générales, et des monographies consacrées à l'auteur. 
mots, des structures et des images, à une époque qui voit - c'est le sujet même de ces textes - l'idée de sens et de vérité textuelle dévalorisée.

\section{Critique des nazis par la raison et l'argumentation : limites d'une stratégie}

Dans sa trilogie romanesque Die Wartesaal-Trilogie 3 , Lion Feuchtwanger adopte une stratégie discursive très claire à l'encontre des nazis : même si, bien entendu, les trois romans dont il est question ne peuvent se résumer à cet aspect, ils présentent le point commun de chercher à démontrer par le rire et la moquerie l'incompétence des nouveaux maîtres de l'Allemagne; ces derniers y sont constamment dénoncés parce qu'ils sont des clowns grotesques et de mauvais politiques, dont le seul argument semble être la violence, verbale et physique.

Dans les lignes qui suivent, on ne reviendra pas sur cet aspect bien connu des œuvres romanesques de Feuchtwanger durant son exil en France. On se bornera à rappeler que ses arguments, en tant que romancier et citoyen, sont, sur ce point, purement rationnels; le rire qu'il cherche à provoquer obéit à une certaine logique. Cela correspond à ce que font Heinrich $\mathrm{Mann}^{4}$ ou Brecht, pour ne citer que les exemples les plus connus d'auteurs en exil qui tentent d'invalider le régime hitlérien par une stratégie discursive fondée sur le rire. Or, la question se pose progressivement de déterminer l'efficacité d'une telle stratégie, qui consiste à attaquer les nazis par le rire, et en ayant recours aux armes rationnelles de l'argumentation : le régime hitlérien est présenté comme ridicule, arguments à l'appui, mais cela n'est pas toujours suffisant. Brecht met en garde les partisans d'une telle tactique discursive lorsqu'il commente Die Brüder Lautensack de Feuchtwanger dans son journal :

[...] scheint mir die feuchtwangersche konzeption weder vom propagandistischen noch vom historischen standpunkt aus sinnvoll. Man bekämpft hitler nicht, wenn man ihn als besonders unfähig, als auswuchs, perversität, humbug, speziell pathologischen fall hinstellt $[\ldots]^{5}$.

Le reproche exprimé ici peut surprendre de la part de l'auteur de La résistible ascension d'Arturo Ui. Car dans sa pièce de théâtre aussi, Hitler et ses affidés sont présentés, on le sait, sous les traits de voyous incultes et faibles, avant qu'un acteur professionnel ne montre au gangster comment faire impression sur les foules par son geste, sa tenue et son verbe. Brecht critique donc ici une stratégie qu'il considère comme vaine. Selon lui, le diagnostic posé par Feuchtwanger et d'autres est juste, qui consiste à dénoncer la faiblesse psychologique des nazis et leur propension à jouer leur propre rôle pour pallier leur incompétence et la cacher sous l'apparence de la force. Mais il ne suffit pas de dire les choses pour détruire le mal. Dès lors

3. Lion Feuchtwanger, Erfolg, Frankfurt a. M., Fischer TB 1650, 1975 ; Die Geschwister Oppermann, Frankfurt a. M., Fischer TB 2291, 1981; Exil, Frankfurt a. M., Fischer TB 2128, 1979.

4. Pour ne mentionner que le seul Heinrich Mann, on rappellera son roman Lidice, de 1942, ou ses essais antinazis parus lors de son exil en France; les nazis y sont raillés systématiquement et y sont présentés comme de mauvais clowns, ridicules et méprisables.

5. Bertolt Brecht, Arbeitsjournal, t. I, 1938-1942, éd. par W. Hecht, Frankfurt a. M., 1973, p. 380. 
qu'Hitler est au pouvoir, une telle stratégie est caduque. Sans doute Brecht a-t-il raison de pointer ainsi les limites d'une telle démarche et, plus largement, les limites de la satire littéraire. Cependant, la lecture détaillée du roman Die Brüder Lautensack et d'autres textes écrits par Feuchtwanger durant l'émigration révèle que Brecht n'a pas vu ou pas voulu voir que le propos de son collègue consiste en réalité justement à mettre en scène les limites de sa propre démarche d'auteur et qu'il ne se contente pas de critiquer le régime par la seule argumentation ${ }^{6}$. Et que, finalement, son ambition est de mettre l'accent sur ce qui fait la spécificité de ce nouveau régime, sur le plan psychologique et philologique : sa propension au mysticisme et à l'irrationnel, qualités présentées par les nazis comme le cœur de l'âme allemande?

En d'autres termes, Feuchtwanger ne répète pas dans Die Brüder Lautensack les situations ni les idées qu'il avait exposés dans la Wartesaal-Trilogie. Bien entendu, la fable, la diégèse du roman de 1942 est semblable aux précédents. Les nazis y sont avant tout ridiculisés. S'ajoute cependant à cette première dimension une autre strate discursive, puisque le romancier dénonce cette fois, en plus, la propension des nazis à manipuler la population en utilisant sa peur et son penchant pour l'irrationnel. En outre, si dans les romans précédents Feuchtwanger attaque les nazis en soulignant prioritairement leur caractère d'escrocs, de menteurs et de comédiens $\left(\right.$ Komödianten $^{8}$ ), désormais, son analyse a évolué et il montre que les nouveaux dirigeants allemands sont devenus des professionnels. C'est leur maîtrise des discours et de la rhétorique qui est le sujet du roman Die Brüder Lautensack, tout autant que leur manque d'épaisseur psychologique et leur violence barbare. Hitler, qui apparaît directement en tant que personnage dans le roman, est certes toujours risible, mais il est surtout terrifiant car il domine son sujet et sait parfaitement manipuler son auditoire, à l'instar du devin mystique Oskar Lautensack, lequel est, on le sait, la figuration romanesque non équivoque du mage Hanussen.

\section{Les nazis ou la manipulation des masses par l'irrationnel et le mythe}

Dans Die Brüder Lautensack, le propos se déplace donc nettement vers une critique de l'irrationnel, tel qu'il est utilisé par les nazis. Feuchtwanger cherche à démontrer

6. Sur ce point, on consultera, pour plus de détails sur les procédés utilisés par Feuchtwanger, l'étude suivante : Frédéric Teinturier, «Die Geschwister Oppermann von Lion Feuchtwanger. Ambivalenz und Philologie ", in Feuchtwanger und Berlin, in Geoffrey V. Davis (dir.), Oxford/ Bern/ Berlin, Peter Lang (Feuchtwanger Studies vol. 4), 2015, p. 39-57.

7. Le propos n'est pas ici de revenir sur l'image de la civilisation allemande dans les discours nazis, mais dans tous les romans de Feuchtwanger, les nazis chantent l'âme éternelle allemande, fière, vraie, droite.

8. La comparaison, bien connue, entre les nazis et de mauvais acteurs, s'inscrit dans un schéma culturel passé, puisqu'il est indirectement tiré de l'analyse nietzschéenne de la Décadence en général, et de la personnalité de Wagner, en particulier. Avec Die Brüder Lautensack, Feuchtwanger change en quelque sorte de paradigme : son analyse du nazisme conserve cette dimension clownesque et théâtrale car son objet n'a pas changé sur ce point; mais il adapte son propos à ce qu'il voit désormais, en décalant le centre de son attaque sur l'irrationnel et le mysticisme. 
que le peuple allemand est victime non seulement d'une escroquerie de la part des nazis, qui se présentent comme les sauveurs de la patrie, mais surtout d'un accès de mysticisme qui le rend vulnérable aux pires manipulations. Hitler est présenté lors de sa première apparition dans le roman comme " réceptif au mysticisme ${ }^{9}$ ». Dès lors, on comprend que ce qui n'était qu'une remarque accessoire dans les romans précédents de Feuchtwanger devient l'essentiel : le romancier entreprend cette fois une critique du national-socialisme à travers ce penchant au mysticisme. Et on réalise qu'une fois de plus, comme dans les romans de la Wartesaal-Trilogie, le propos de l'auteur est de nature éminemment philologique. Le roman est d'ailleurs précédé par quelques pages dans lesquelles le narrateur introduit le sujet de l'histoire et en définit les enjeux : en réalité, la première page est consacrée à la présentation d'Oskar Lautensack, tel qu'il est, misérable, avant de connaître une fulgurante ascension sociale. Mais rapidement, le récit est interrompu par la voix auctoriale ${ }^{10}$ qui annonce son intention de dénoncer dans les pages qui suivent l'« occultisme » nazi, en apportant trois preuves ${ }^{11}$. Son point de vue est donc indubitablement celui du scientifique, du philologue, comme dans ses autres romans sur le nazisme. Et le narrateur conclut sa remarque en se plaçant ironiquement sous le patronage de Kant :

Schon Immanuel Kant hat sich wehren müssen gegen die Art, wie Hellseher und ihre Anhänger seinen Bericht über Swedenborg ausnützten. In einem hübschen, kleinen Buch 'Träume eines Geistersehers' rückt er von diesen Fanatikern $a b^{12}$.

Malgré tout, on voit que le propos, dans le roman Die Brüder Lautensack, est avant tout de nature défensive; en représentant ainsi des écrivains et des penseurs, des professionnels de l'écrit en général, et du récit en particulier, Feuchtwanger entreprend dans son nouveau roman de contre-attaquer ceux qui « utilisent » contre lui et ses personnages leurs assertions. Les temps ont changé, nous dit ainsi Feuchtwanger, mais c'est aussi une manière de signaler à ses lecteurs de 1943 qu'à ses yeux, le danger occulte n'est que la répétition d'autres dangers antérieurs. Rien de plus, pourrait-on ajouter.

Dans Die Brüder Lautensack, il s'agit donc de dénoncer les mauvaises interprétations volontaires, les tromperies conscientes par mensonge, les fausses preuves apportées par les « mages » à l'appui de leur tentative pour faire accroire au public la véracité de leurs exploits mystiques. Quelques exemples, parmi l'accumulation impressionnante des «Zeugnisse » que nous livre le romancier, révèlent que son point de vue est bel et bien de nature philologique : il s'agit pour lui de révéler les manipulations de la vérité par les nazis, qui sont ainsi, en quelque sorte, les premiers partisans des «fake news » et de la «post-vérité ${ }^{13} »$.

9. Lion Feuchtwanger, Die Brüder Lautensack, Berlin, Aufbau, 1994, p. 44 : « empfänglich für Mystik ».

10. Die Brüder Lautensack, p. 8 sqq.

11. "Zeugnisse », preuves qui ressortent d'une analyse et soutenues par des éléments tangibles : « Material », faits avérés.

12. Ibid., p. 13.

13. Notons que le personnage du mage, Oskar Lautensack, se présente sous les traits d'un escroc, d'un «magicien ». D'ailleurs, lors de ses performances de divination, il est toujours accompagné et aidé de son assistant Alois Pranner, prestidigitateur de son état, et dont le surnom est Cagliostro. Ce dernier 
Oskar Lautensack, qui est comparé à Orphée, au devin Kalchas ${ }^{14}$ ou au magicien Klingsor ${ }^{15}$, se caractérise donc par sa capacité, bien réelle, quoiqu'inexplicable, à « voir » les choses, à prévoir des événements qui se dérouleront dans la vie des gens qu'il soumet, la plupart du temps lors d'une séance publique, à son examen. Ces «prévisions » sont de deux natures. Le premier cas de figure est le suivant : ce qu'annonce Oskar est très vague, trop imprécis, si bien que les personnes concernées peuvent considérer a posteriori la prédiction comme véridique, une fois qu'un événement pouvant être interprété comme étant celui annoncé, intervient. C'est le cas le plus fréquent. Son degré d'efficacité pour convaincre la foule de la réalité des pouvoirs divinatoires du mage est relativement faible, mais il repose sur le caractère public de la prédiction : l'adéquation entre un événement précis (bonne fortune, revirement politique majeur, ...) et sa prétendue annonce par le mage n'est pas validée par la seule interprétation individuelle du patient à qui Oskar a fait son annonce. Car une telle interprétation nécessite, quoi qu'on en dise, et quel que soit l'état de crédulité ou de réceptivité de la personne, le recours aux fonctions rationnelles de base. L'habileté du mage consiste dans le fait qu'il ne laisse pas l'individu concerné seul juge de la véracité de sa prédiction; en effet, il fait son annonce en public et c'est la réaction unanime et immédiate de la foule, réaction qui mêle les marques d'assentiment et d'admiration face au caractère audacieux de la prédiction, qui prédispose l'ensemble du public à reconnaître le pouvoir du mage, sans même qu'il soit besoin de preuves, puisque tout le monde a bien vu et bien dit, unanimement, combien la prédiction est vraie ou pourrait l'être ${ }^{16}$.

Le second cas de figure est plus problématique car il repose sur une réelle prise de risque en direct d'Oskar. Lors de la représentation, il a recours à une technique de manipulation psychologique dite « technique de suggestion ${ }^{17}$ ». Comme il connaît le public qui se trouve devant lui - son assistant s'est renseigné sur chacun des présents, alors que le mage est censé ignorer l'identité des invités -, Oskar peut s'adresser à une personne particulière de l'assemblée en connaissance de cause. Il connaît les problèmes que rencontre telle personne, sur le plan personnel, ou professionnel, il est au fait de son état d'esprit et de ses inquiétudes du moment. Il utilise donc ce savoir préalable pour orienter dans la direction qu'il souhaite les questions qu'il pose et, surtout, les réponses qu'on lui fait lors d'une séance de « télépathie » et c'est lui, cette fois, qui se place dans la position de l'interprète : au gré des réponses, du ton de celles-ci, Oskar parvient à suggérer à la personne qui se trouve face à lui, isolée

prépare le terrain, sonde le public avant l'entrée en scène du maître; il repère les spectateurs les plus susceptibles d'être manipulés. Lautensack est donc un menteur, un magicien de pacotille, un homme de théâtre, art dont il sait utiliser les effets. Cependant, à la différence de tous les autres personnages de Komödianten dans l'œuvre de Feucthwanger, Oskar est réellement un devin. Le narrateur le montre entrer en transe, avoir des crises, des « visions », et certaines de ses interventions en public ne sont pas explicables rationnellement. En outre, le devin, qui n'est pourtant pas dupe de ce qu'il fait, se laisse parfois prendre à son propre jeu et le narrateur parle plusieurs fois d' " autopersuasion ». Cf. Die Brüder Lautensack, p. 123-125.

14. Die Brüder Lautensack, p. 201.

15. Die Brüder Lautensack, p. 219.

16. Entre autres exemples : Die Brüder Lautensack, p. 191.

17. Die Brüder Lautensack, p. 80 : «Suggestivtechnik ». 
du reste l'assemblée, qu'elle pourrait avoir pensé, plus tôt, à telle ou telle chose. Si bien que finalement il se met à manipuler la personne, et surtout, le public spectateur, qui est sa véritable cible. Plus d'une fois ${ }^{18}$, l'individu isolé n'est pas entièrement convaincu par ce que le mage révèle, mais il est déstabilisé et ne peut nier ce qui est dit. C'est l'attitude hésitante, confuse de cette personne, que le reste du public interprète comme signe tangible qu'une vérité vient d'être révélée.

On le voit, la conversion des sceptiques n'est pas le but principal du devin Oskar, ni des nazis d'ailleurs. Leur stratégie vise à atteindre une conversion collective et pour le moins l'assentiment de la foule. Et leur moyen d'action est celui de l'émotion (peur, enthousiasme, admiration...), qui est bien plus prévisible et facile à orienter que l'individu, qui sera toujours tenté de raisonner. Jouer ainsi sur le trouble et, plus largement, sur les émotions, est bien plus efficace et c'est ainsi, dans un second temps, que le devin complète son action de manipulation. Après avoir convaincu la foule, il peut, lors d'une séance individuelle, manipuler un individu qui aurait assisté à une séance collective; ce dernier, ou cette dernière, puisqu'il s'agit le plus fréquemment de femmes - et d'Hitler lui-même - est déjà réceptif car il est encore sous le coup de l'émotion. C'est le cas de Käthe, la sœur du journaliste politique Paul Cramer, lequel est, dans le roman, le représentant du logos et critique les nazis par la démonstration. La jeune femme finit par être la maîtresse d'Oskar, mais ce sont les qualités d'acteur du mage qui l'ont fascinée en premier lieu ${ }^{19}$. Et son frère, Paul, qui entreprend, tel un double du romancier, de déconstruire méthodiquement, dans ses articles de presse, le mode de manipulation mentale utilisé par les nazis et Oskar Lautensack, finit par admettre l'inefficacité de sa critique avec les armes de la rationalité. Dans une lettre à sa sœur, il cite en effet au sujet d'Oskar ces lignes de Goethe, dans un geste culturel qui achève de faire de lui le symbole de la philologie à l'œuvre face au nazisme :

\footnotetext{
Am furchtbarsten », zitiert er, « erscheint das Dämonische, wenn es in irgendeinem Menschen überwiegend hervortritt. Es sind nicht immer die vorzüglichsten Menschen, weder an Geist noch an Talent. Aber eine ungeheure Kraft geht von ihnen aus, und sie üben eine unglaubliche Gewalt über alle Geschöpfe. Vergebens, dass der hellere Teil der Menschen sie als Betrogene oder als Betrüger verdächtig machen will, die Masse wird von ihnen angezogen ${ }^{20}$.
}

Face à ceux qui manipulent le public au moyen de forces irrationnelles, la rationalité n'a donc plus de prise. Et le résultat de l'opération, qui ne fait aucun doute dès le début du roman, est mis en scène par Feuchtwanger comme la défaite de l'interprète, de l'argumentation, de la recherche de la vérité. Le personnage de Paul Cramer sera « suicidé » par les hommes de Proell - qui est la figuration romanesque de Röhm. Et il est signifiant que dans Die Brüder Lautensack on puisse remarquer un glissement sémantique à propos de la vérité : de valeur-repère, au singulier, elle devient un terme pluriel, il y a désormais plusieurs vérités et, surtout, elle se voit adjoindre

18. Voir la scène chez Ilse Kadereit, p. 82 et aussi p. 121 et suivantes.

19. Die Brüder Lautensack, p. 177.

20. Ibid., p. 167. 
divers qualificatifs : Oskar diffuse progressivement, au fur et à mesure de ses séances d'occultisme, l'idée qu'il existe une « vérité supérieure ${ }^{21} »$.

\section{La littérature face aux cochons : les insuffisances du philologue et celles du public}

Quoi qu'il en soit, le message délivré par Feuchtwanger dans ce roman est qu'il faut désormais entériner la défaite du discours rationnel. La démonstration des torts et des insuffisances des nazis ne suffit plus à valider la critique qu'on leur oppose. Au contraire, puisque c'est le discours rationnel dans son entier qui est disqualifié dans cette période où triomphe le discours irrationnel et ses avatars, comme le mythe.

Le discours philologique se définit comme celui qui, dans les différents domaines de la pensée où il est à l'œuvre - l'histoire, la littérature, la philosophie - a recours à la présentation ordonnée d'arguments, à la reconnaissance de la valeur d'éléments factuels contre les simples opinions subjectives et, partant, à la mise en valeur des vertus de l'interprétation rationnelle contre l'émotion et l'irrationnel. Or si ce discours est disqualifié, la cause n'en incombe pas, chez Feuchtwanger, uniquement à la force de ses adversaires qui, nous venons de le rappeler, sont de piètres personnes. La richesse souvent sous-estimée des romans de l'exil écrits par Feuchtwanger provient en grande partie de l'ambiguïté constante qui entoure les partisans du rationnel. Les personnages qui défendent les valeurs de l'auteur sont eux aussi présentés sous un jour problématique, et il est fréquent que leurs défauts et leurs insuffisances contribuent à favoriser la victoire de leur adversaire. Ce trait récurrent propre à Feuchtwanger, cette ambiguïté constitutive de son art romanesque, sont connus ${ }^{22}$, si bien qu'il n'est pas nécessaire de s'y attarder. Nous nous bornerons à souligner que, comme dans la Wartesaal-Trilogie, le personnage du philologue est critiquable dans Die Brüder Lautensack: Paul Cramer est colérique, très jaloux de sa sœur, et ses motivations pour attaquer le mysticisme d'Oskar, l'amant de sa sœur, sont ambiguës. Surtout, Paul est parfaitement conscient des insuffisances de ses armes philologiques et rationnelles pour contrer le danger mystique des nazis, mais il semble ne pas vouloir s'adapter et il persiste dans la voie qu'il a initialement choisie : écrire des articles dans lesquels il déconstruit méthodiquement le mythe hitlérien. En outre, comme dans Die Geschwister Oppermann, on montre le personnage rationnel obsédé par le style d'Hitler - signe d'irrationnel et d'émotion inefficace. Ce dernier écrit un mauvais allemand et il s'agit de le dénoncer avant toute chose ${ }^{23}$. Or Paul est bien conscient du fait que si son article sur le style du Führer est efficace auprès des autres philologues, il n'arrive pas à toucher le public. Surtout, cette attaque a pour conséquence de provoquer la réaction irrationnelle d'Hitler, qui ne prend au

21. Ibid., p. 176 : Alois, l'assistant prestidigitateur, n'est pas dupe, bien entendu, et ne supporte plus, dans la bouche de son employeur Oskar, cette prétention à ne pas être qu'un escroc; il lui lance alors : « Leck mich am Arsch mit deiner höheren Wahrheit. »

22. Note 4.

23. Die Brüder Lautensack, p. 165 sqq. 
sérieux cet opposant qu'à partir du moment où ce dernier l'attaque sur le domaine linguistique. Ce qui provoquera directement la perte de Paul.

Au-delà de cet aveuglement du personnage dont on peut légitiment penser qu'il est le plus proche du point de vue du romancier et qu'il en est le plus souvent le porteparole, il est une nouvelle dimension mise en avant dans Die Brüder Lautensack, et qui sera dès lors récurrente dans plusieurs autres écrits de Feuchtwanger : ce n'est plus seulement du producteur de la critique antinazie dont il s'agit de souligner les faiblesses, mais du récepteur, c'est-à-dire du public allemand. Si les nazis peuvent aussi librement user de l'irrationnel et de l'occulte pour s'imposer, c'est parce que la population allemande des années 1930 est peu encline à réfléchir et qu'elle se laisse abuser bien facilement. Le roman peut ainsi également se lire comme un règlement de compte avec le public allemand. En décryptant les mécanismes de la manipulation, Feuchtwanger dénonce donc autant le manipulateur que le manipulé. On en trouve de nombreux exemples dans Die Brüder Lautensack; parmi eux, on retiendra le fait que toutes les classes sociales semblent être susceptibles de succomber aux charmes d'Oskar, et aux effets de son art occulte. Aussi bien le petit-bourgeois que le grand industriel, qui est, dans l'intrigue romanesque, le cœur de cible des dirigeants nazis au moment d'accéder au pouvoir; c'est d'ailleurs la raison pour laquelle Hitler et ses affidés s'intéressent aux talents mystiques et pseudo-télépathiques du mage voyant.

Un autre texte de Feuchtwanger permet d'aller plus loin sur ce point, car il thématise lui aussi l'effet de l'irrationnel et du mythe sur le public encore plus nettement que dans la Wartesaal-Trilogie et Die Brüder Lautensack. Il s'agit de la nouvelle Odysseus und die Schweine oder das Unbehagen an der Kultur, qui a été écrite juste après la Seconde Guerre mondiale ${ }^{24}$. Il n'est plus question des nazis, pas même métaphoriquement, mais directement du mythe et du rapport entre vérité factuelle et littéraire. Ce court récit tardif, sous-estimé et jamais véritablement analysé, est plus riche d'enseignement qu'il paraît ${ }^{25}$. Le sujet que Feuchtwanger s'amuse à y imaginer

24. Odysseus und die Schweine oder das Unbehagen an der Kultur, in Lion Feuchtwanger, Die große Passion des Klavierspielers Morgenroth, Erzählungen, Berlin, Aufbau Taschenbuch, 1998, p. 348373. La nouvelle a été écrite en 1947, avant de paraître en 1948 dans la revue Ost und West dirigée par Alfred Kantorowicz, puis au sein d'un recueil en 1950.

25. Le lecteur est naturellement amené à se demander si Feuchtwanger instaure dans ce récit, d'une manière ou d'une autre, un dialogue avec Dialektik der Aufklärung de Horkeimer et Adorno, paru en 1944. (écrit entre 1939 et 1944, ce recueil d'essais est rendu public en 1944, avant une première édition sous forme de livre en 1947 : Max Horkheimer, Theodor W. Adorno : Dialektik der Aufklärung, Amsterdam, Querido, 1947).

En effet, le chapitre 2 de Dialektik der Aufklärung s'intitule, comme on le sait, Odysseus oder Mythos und Aufklärung. En outre, le sujet traité par l'écrivain littéraire semble proche de celui abordé par les philosophes : Ulysse est utilisé dans les deux cas comme symbole de la civilisation occidentale rationnelle et, dans les deux textes, on peut lire une réflexion critique sur la notion de mythe. Dans la mesure où le recueil des deux philosophes a connu, dans le cercle culturel germanophone, le retentissement que 1'on sait et que, surtout, Feuchtwanger et Adorno se fréquentaient durant l'exil en Californie et étaient voisins, il semble difficile de penser que les deux textes soient totalement étrangers l'un à l'autre; cela étant dit, l'étude de leur rapport - étude qui, pour aboutir, devrait faire l'objet d'une analyse séparée - ne représenterait pas un apport décisif dans le cadre de la présente étude, qui se consacre à l'univers de Feuchtwanger, tel qu'il est constitué dans ses textes. En outre, les limites d'un tel rapprochement apparaissent d'emblée : là où les deux philosophes pointent le rapport 
permet d'explorer les confins entre l'histoire, le récit historique et sa véracité, le mythe oral, les modalités de sa transmission et la littérature écrite fixée par un scribe. La nouvelle se présente comme une sorte de coda à l'Odyssée d'Homère; Ulysse vieillit à Ithaque et s'ennuie. Surtout, il réfléchit au temps qui passe et à la véracité de ses souvenirs par rapport aux récits qui sont faits de ses exploits. Peu satisfait du manque d'adéquation entre les faits réels tels qu'ils se sont déroulés à son retour à Ithaque et la façon dont les poètes (tous sont des 'Homères', terme qui est devenu un nom de métier et une fonction) l'ont transcrite en un mythe dont tous savent qu'il n'est pas exact (notamment pour ce qui est du nombre de prétendants tués), Ulysse pense avec nostalgie à son séjour chez les Phéaciens. Il décide d'y retourner. Le roi Alcinoos est aimable, mais visiblement peu content de revoir ce roi grec, dont il sait bien, lui, que les exploits chantés ne correspondent pas à la réalité. La civilisation des Phéaciens est présentée comme une utopie, c'est un pays supérieur en tout, surtout sur le plan technique, et qui désire rester à l'écart des guerres. La civilisation phéacienne a découvert l'usage du fer et de l'écriture, qu'elle ne destine cependant pas à la transcription des mythes et des récits des 'Homères'. L'écriture est réservée aux choses sérieuses, économiques et stratégiques. Les Phéaciens ont eux aussi un poète officiel, un 'Homère', dont les chants remplissent l'âme d'Ulysse de nostalgie. Un dialogue entre le poète des Phéaciens et le roi d'Ithaque commence; Ulysse finit par avouer à ce poète étranger qu'il n'a pas dit toute la vérité concernant ses aventures. Il revient en particulier sur l'épisode avec Circé. Le récit littéraire, fidèle aux récits d'Ulysse, affirme, on le sait, que ce dernier a pu défaire le sortilège qui avait transformé ses compagnons en cochons. En vérité, avoue Ulysse, ces derniers n'avaient pas envie de redevenir des hommes, ils étaient bienheureux en animaux, délestés du poids de leurs préoccupations. On comprend donc, à la fin de la nouvelle, que la raison du malaise d'Ulysse provient du manque de vérité de tout récit par rapport aux faits, mais que surtout, c'est le caractère inavouable de ces faits qui lui posent le plus problème : ses compagnons ont préféré rester des cochons! Ironique, la pointe de la nouvelle contient cependant une signification métaphorique essentielle : les compagnons qui préfèrent rester des cochons sont les premiers spectateurs de ses exploits, ce sont eux qui figurent en quelque sorte le public lecteur-auditeur du spectacle de l'Odyssée; or ce public premier ne ressent aucune envie de continuer son rôle ni d'accompagner le héros dans ses exploits, ou de continuer à l'écouter. Feuchtwanger se montre ainsi très dur envers la figure du lecteur, de la population, ou encore des gens ordinaires, bref : avec les récipiendaires de la littérature, entre acteurs secondaires et spectateurs passifs des aventures des protagonistes. Ulysse se voit ainsi contraint de falsifier la vérité historique pour éviter que son récit, qui servira de matrice à ceux d'Homère, ne devienne ridicule. Sans les compagnons, en effet, plus aucun retour n'est possible, car ces adjuvants sont nécessaires pour manœuvrer le navire. En d'autres termes, la nouvelle Odysseus présente le destin de

de l'Occident au discours mythologique, l'écrivain littéraire se concentre sur son domaine, à savoir la réflexion philologique; il s'intéresse ainsi exclusivement aux mécanismes d'élaboration du texte, et il thématise le moment où le récit oral est transcrit par le poète et devient un écrit transmissible. Son propos repose donc sur l'étude du texte en tant qu'objet, son sens, son interprétation, et ne met pas en place de réflexion sur la notion de mythe dans une perspective philosophique ou civilisationnelle. 
la littérature comme étant dépendant des boétiens, de ce public récalcitrant et gêneur, qui se laisse aisément aller et qui ne rêve qu'à se prélasser au soleil dans la boue plutôt que d'être partie prenante dans les exploits du rusé Ulysse.

\section{Une réflexion sur le mythe et le fonctionnement du mythologique}

Au-delà de l'ironie cruelle, la nouvelle en question a comme sujet profond le rapport entre récit et réalité, entre littérature et faits. Il est ici essentiel de distinguer, nous dit Feuchtwanger, entre littérature écrite et mythe oral. Toute la première partie de la nouvelle doit ainsi se lire comme une réflexion sur le mythe, la poésie mythologique et leur rapport à la réalité historique, celle qui est vécue par le témoin des faits.

Dans cette nouvelle, Feuchtwanger reprend, en les approfondissant, les thématiques qui sont les siennes dans la plupart de ses essais sur le roman et le roman historique en particulier. Ce qui l'intéresse au premier chef, comme dans Die Brüder Lautensack, ce n'est pas la véracité prétendue de tel ou tel exploit d'Ulysse la nouvelle met en avant à plusieurs reprises la faiblesse du souvenir, voire le peu de fiabilité du témoin et le travail du temps sur sa mémoire. Ce qui intéresse l'écrivain Feuchtwanger, c'est avant tout l'effet produit, c'est le résultat final, une fois que le poète s'est saisi du récit oral fait par le témoin des événements. Ce point de passage entre réalité et poésie, tel qu'il est mis en scène dans la nouvelle, permet d'intenter un rapide procès à l'idée de vérité. Odysseus und die Schweine est un texte construit sur un glissement du concept de vérité ${ }^{26}$ jusqu'à celui de « vraisemblance ${ }^{27}$ ». La qualité du poète se mesure à sa capacité à transformer en vérité éternelle, par le mythe oral qui fait grand effet sur le public, ce qui n'est ni vrai ni éternel. Et le critère décisif, qui préside au choix des éléments qui seront retenus dans le chant d'Homère, n'est pas uniquement politique ou politiquement correct, même si la fin de la nouvelle semble privilégier cette idée ${ }^{28}$; le critère premier est de nature esthétique et philologique : on retient ce qui est crédible, ce que le public pourra croire, ce qui est réussi. La métaphore utilisée par le poète des Phéaciens va dans ce sens : les faits relatés par Ulysse sont le squelette, mais le corps entier, visible du mythe, est créé par le poète ${ }^{29}$. La vérité, à condition qu'elle ne soit pas contreproductive, comme dans le cas des cochons, n'est qu'un élément parmi d'autres, chronologiquement le premier, mais pas le plus décisif. Ces réflexions semblent bien peu originales, en vérité, n'était l'ironie du nouvelliste qui s'amuse à montrer à son lecteur les coulisses de la naissance du mythe. Pourtant, le serpent se mord la queue, et c'est ce qui fait tout le sel de l'exposé métapoétique présenté dans cette nouvelle. En effet, si le premier critère pour retenir tel élément narratif plutôt qu'un autre, est la capacité du

26. Odysseus und die Schweine, p. 351. La vérité et sa recherche sont les motivations d'Ulysse.

27. Ibid., p. 366 : « Glaubwürdigkeit».

28. On préfère passer sous silence la vérité sur les hommes qui ont préféré rester des cochons, sans doute pour ne pas créer des vocations.

29. Odysseus und die Schweine, p. 369-370. 
public à l'accepter, ou à le trouver « vraisemblable», comment accorder cela avec le fait que ce public, justement, est décrit dans ce texte comme un ramassis d'imbéciles et d'hommes qui préfèrent rester métamorphosés en cochons? Plus rien ne semble donc fiable, et voilà sans doute le message amusé de Feuchtwanger. En mettant l'accent comme il le fait sur le récipiendaire du texte poétique, et en premier lieu sur ses insuffisances récurrentes, l'auteur refuse à tout texte, quel qu'il soit, même les plus emblématiques de l'histoire littéraire occidentale - La Guerre de Troie et L'Odyssée - la possibilité d'avoir un statut définitif et intouchable. Le lecteur se voit refuser quant à lui le droit d'avoir son mot à dire. Les deux seuls habilités à juger de la qualité littéraire d'un texte sont le poète et Ulysse. Et encore, ce dernier se voit-il reconnaître ce droit non pas parce qu'il est le héros des événements, mais parce qu'il est « avide de savoir » et « rusé », les deux épithètes homériques les plus fréquentes accolées au roi d'Ithaque dans la nouvelle.

Mais l'entreprise de déconstruction de Feuchtwanger ne s'arrête pas là, car il nous montre Ulysse vieillissant, fatigué et plus très sûr de ne pas vouloir, lui aussi, terminer sous l'apparence d'un cochon. Finalement, on est même en droit de s'interroger sur le rôle du poète; cet Homère prototypique, dont il importe peu de savoir le nom, est le seul juge, finalement, mais il n'existe pas. Il a beau être plus sage qu'Ulysse lui-même ${ }^{30}$, il n'est qu'un principe, pas une personnalité. Chaque royaume a son Homère. Il ne reste donc que le texte. Mais le statut même du texte poétique est ici problématique : sans doute faut-il d'ailleurs voir dans cette déconstruction du sens de la poésie et dans l'incertitude ironique de son statut l'intérêt de la nouvelle de Feuchtwanger.

Comme dans Die Brüder Lautensack, le romancier nous invite donc en réalité à nous interroger sur la nature du texte littéraire, sur son statut social, sur sa valeur. Dans le roman de l'exil, ce texte est fragile, car les auteurs sont persécutés et le public manipulé. Les articles de Paul Cramer n'existent que parce qu'ils sont lus; dès qu'ils ne le sont plus, la critique disparaît. Dans Ulysse et les cochons, le texte est extrêmement fragile, car Feuchtwanger saisit le moment de l'histoire de la civilisation occidentale où le texte poétique effectue ou pourrait effectuer le passage de l'oralité à l'écrit. En effet, Feuchtwanger se plaît à laisser planer le doute sur la valeur de la découverte de l'écriture par les Phéaciens. Certes, ces derniers utilisent l'écriture pour fixer l'oralité, mais pas pour les chants de leur Homère, le roi plaisantant même avec Ulysse qui avait demandé s'ils comptaient mettre par écrit ses exploits passés. Non, l'écriture est réservée aux choses sérieuses, commerciales ${ }^{31}$. La poésie se voit reléguée par la civilisation utopique et supérieure des Phéaciens au rang d'activité sociale subalterne et réduite à son caractère de divertissement. Aucune valeur historique ou sociale ne lui est reconnue, semble-t-il. En d'autres termes, pour reprendre l'opposition qui est ainsi mise en place, la littérature est condamnée à rester un mythe, certes figé par les chants de l'Homère qui a écouté Ulysse et qui a décidé ce qu'il allait garder des récits du héros, mais un texte oral, auquel on refuse la noblesse de l'écriture. Dans le schéma mis en place par l'auteur

30. Ibid., p. 366.

31. Ibid., p. 367. 
dans cette nouvelle tardive, le poétique est, très sérieusement cette fois, mis en scène comme ce qu'il y a de plus fragile. Du côté de sa production, d'une part, laquelle est présentée comme aléatoire et peu en rapport avec une quelconque valeur de vérité préalable; du côté de sa réception, d'autre part, car le public, Ulysse compris, est considéré comme peu fiable.

Après une lecture croisée d'Ulysse et les cochons et de Die Brüder Lautensack, force est de constater que c'est la même fragilité du poétique qui est en réalité le fondement du propos auctorial dans le roman sur les nazis et leur rapport au mystique. Le poète - ou plus précisément, le philologue, l'écrivain interprète - est plus que jamais soumis à la violence de son époque; le public est à l'image des compagnons d'Ulysse : bienheureux dans sa fange; et la transmission, la pérennité du texte lui-même - question dont la nouvelle sur Ulysse révèle l'importance pour Feuchtwanger - est problématique. En effet, dans l'Allemagne hitlérienne, on opère un retour en arrière inédit, qui figure peut-être le point nodal de toutes les critiques adressées au nouveau régime par l'intellectuel Feuchtwanger : c'est une nouvelle civilisation qui se met en place à partir de 1933, au sens où l'écrit se voit refuser l'importance qui est la sienne depuis l'Antiquité. Les nazis, tels qu'ils sont présentés dans le roman, opèrent un retour à l'oralité, et ils n'accordent de crédit qu'au verbe - ce qui ne signifie pas : à la parole donnée -, c'est-à-dire à l'effet produit par la proclamation devant un public qu'il s'agit non de convaincre mais de captiver. L'opposition, la dernière résistance contre cette nouvelle civilisation, face à cette contre-civilisation, est figurée par l'écrivain, celui qui a encore foi dans le texte écrit par rapport au texte oral. Ce que détruit Hitler en réinstaurant la primauté de l'oral sur l'écrit, du spectacle collectif sur l'acte de lecture individuel, c'est la possibilité du travail d'interprétation et d'exégèse, la possibilité de mettre en place une réflexion rationnelle sur un texte de pouvoir. Le discours hitlérien, entièrement oral, est destiné à produire un effet irrationnel sur la foule, et il se refuse à l'analyse philologique (sauf en cas d'enregistrement, mais c'est une manière détournée de le fixer par l'écriture). C'est en cela que les nazis, tels qu'ils sont mis en scène dans Die Brüder Lautensack, représentent un danger civilisationnel. Leur propension à l'irrationnel est secondaire; c'est leur rapport au discours et au logos qui est présenté comme le principal problème. Et la trame narrative de ce roman montre la convergence fatidique d'Hitler et du mage Oskar l'un vers l'autre. Le premier est certes de plus en plus réceptif à la volonté du second, mais leur parenté repose bien plutôt sur leur croyance commune aux forces de l'inexplicable et à la primauté qu'ils accordent à l'élan mystique et donc non analysable qui pousse deux êtres l'un vers l'autre. En d'autres termes, Oskar sert narrativement - et au-delà du fait qu'il est le portrait romanesque de Hanussen - à figurer l'attirance des nazis pour le discours mystique et le personnage de Paul Cramer sert a contrario à figurer leur rejet du discours rationnel et argumenté. 
